Abstract

\title{
Acute Effect of Two Different Post-Activation Potentiation Running Protocols on Sprint Performance of Preadolescent Boys ${ }^{\dagger}$
}

\author{
Dimitrios Petrakis *, Eleni Bassa and Anastasia Papavasileiou \\ Laboratory of Evaluation of Human Biological Performance, School of Physical Education and Sport Science \\ at Thessaloniki, Aristotle University of Thessaloniki, 57001 Thessaloniki, Greece \\ * Correspondence: dpetrakis30@gmail.com \\ + Presented at the 9th Greek Conference of Biochemistry and Physiology of Exercise, Thessaloniki, Greece, \\ 18-20 October 2019.
}

Published: 3 September 2019

\begin{abstract}
AIM: Forward and backward sprint are two types of locomotion commonly used in sports. The purpose of the present study was to examine the acute effect of two post-activation potentiation sprint protocols, including the aforementioned types of locomotion, in subsequent 20$\mathrm{m}$ sprint performance (SP) and the possible fatigue caused by them in preadolescent boys. MATERIAL \& METHOD: Fourteen recreationally active preadolescent boys (aged $12.49 \pm 0.52$ years) volunteered to participate in the study. Boys' SP was evaluated randomly on three nonconsecutive days. SP was evaluated 4 min after (a) a typical warm-up (3 min jogging and dynamic stretching), (b) the typical warm-up followed by $3 \times 10 \mathrm{~m}$ forward sprint and (c) the typical warmup followed by $3 \times 10 \mathrm{~m}$ backward sprint. The Microgate Witty Wireless Training Timer was used for the evaluation of SP and the 10-degree OMNI scale for the evaluation of the rate of perceived exertion. RESULTS: ANOVA with repeated measures indicated no statistically significant effect of protocol on the $0-5,0-10$ and $0-20 \mathrm{~m} \mathrm{SP}(p>0.05)$. On the contrary, it was found that the protocols in which $3 \times 10 \mathrm{~m}$ forward or backward sprint was incorporated caused statistically significant greater fatigue than the control protocol $(p<0.001)$, without any differences between them. CONCLUSIONS: The implementation of three 10-m sprints, either forward or backward, to a standard warm-up does not cause acute improvement in 20-m sprint performance in preadolescent boys, as both protocols cause greater fatigue than a typical warm-up. Therefore, their addition to warm-up of preadolescent boys is not recommended.
\end{abstract}

Keywords: preadolescence; post-activation potentiation; sprint performance; fatigue

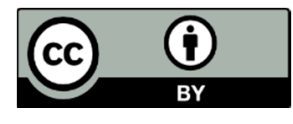

(C) 2019 by the authors. Submitted for possible open access publication under the terms and conditions of the Creative Commons Attribution (CC BY) license (http://creativecommons.org/licenses/by/4.0/). 